\title{
A theory waiting for an experiment: pair-state formation in a nanocrystal
}

\author{
J.F. Suyver ${ }^{\mathrm{a}, *}$, R. Meester ${ }^{\mathrm{b}}$, J.J. Kelly ${ }^{\mathrm{a}}$, A. Meijerink ${ }^{\mathrm{a}}$ \\ ${ }^{a}$ Debye Institute, Physics and Chemistry of Condensed Matter, Utrecht University, P.O. Box 80.000, 3508 TA Utrecht, The Netherlands \\ ${ }^{\mathrm{b}}$ Division of Mathematics and Computer Science, Free University of Amsterdam, De Boelelaan 1081a, \\ 1081 HV Amsterdam, The Netherlands
}

\begin{abstract}
For certain dopants, luminescence measurements allow one to distinguish between single-ion and pair-state dopant emission. In a bulk crystal the concentration of each of these dopant-states can be calculated from the dopant fraction $f$ present in the material, and is found to correlate with luminescence measurements. However, for a nanocrystalline hostlattice, these concentrations have not been calculated due to the difference in coordination numbers for ions at the surface (a substantial fraction in nanocrystals) and in the bulk. Simulations were recently published. Here, the exact solution is presented (though not derived), which allows one to calculate the fraction of dopants that are part of a pairstate for any dopant concentration, crystal size or crystal structure, without having to perform simulations. Several examples are presented and discussed.
\end{abstract}

(C) 2002 Elsevier Science B.V. All rights reserved.

Keywords: Nanocrystal; Pair-state; Probability theory; Simulation

\section{Introduction}

Most scientists interested in luminescence know that the emission characteristics of a dopant ion depend not only on the host lattice, but also on the dopant ion concentration. A famous example is the luminescence of chromium in aluminum oxide. At low $\mathrm{Cr}^{3+}$ concentrations, the emission occurs solely at $693.4 \mathrm{~nm}$. However, at higher $\mathrm{Cr}^{3+}$ concentrations, four additional lines are observed in the wavelength range $698-702 \mathrm{~nm}$. These lines are due to the spin-spin interaction that occurs in

*Corresponding author. Tel.: + 31-30-253-2214; fax: +3130-253-2403.

E-mail address: j.f.suyver@phys.uu.nl (J.F. Suyver).
$\mathrm{Cr}^{3+}$ pairs. ${ }^{1}$ As a result, the groundstate splits into four levels with different energy, which causes the four additional lines to appear in the emission spectrum. Ideally, when one measures the ratio of $693.4 \mathrm{~nm}$ radiation to $698-702 \mathrm{~nm}$ radiation, one finds the ratio of single $\mathrm{Cr}^{3+}$ ions to $\mathrm{Cr}^{3+}$ pairs in the crystal. For bulk crystals, these types of experiments have been performed (albeit for other host-lattice/dopant combinations) and the observed ratio agrees with a straightforward statistical prediction proposed by Behringer [2].

\footnotetext{
${ }^{1}$ Note that the chromium pairs responsible for this emission are not nearest neighbors, but next-nearest neighbors. This has been ignored for simplicity of the example. For a thorough discussion see. Ref [1].
} 
Similar experiments can also be done using doped nanocrystals. These nanocrystals show interesting changes in the electronic structure as the particles become smaller. Synthesis procedures to make very small $(1-10 \mathrm{~nm})$ nanocrystals are well established and fundamental research on the changing properties as a function of particle size has yielded fascinating results [3-7]. The formation of pairs of dopant-ions in nanocrystals can be important for the magnetic and optical properties of these particles [8-10], or for studies on single magnetic nanocrystals [11]. Nanometer-sized materials have a much larger surface area than bulk crystals. As a result, for the same concentration of dopant ions the fraction of pairs will be different for different particle sizes because the coordination number of ions at the surface is lower than that of ions in the bulk. The contribution of dopants at the surface becomes increasingly important as the nanocrystal decreases in size and this complicates the determination of the number of pairs.

This paper will show calculations to find the probability of forming at least one dopant pairstate in the nanocrystal. Also, the fraction of dopants that are part of a pair-state is studied. The calculations are done in two ways: first, through computer simulation of a zincblende crystal, in which the number of isolated dopants and the number of dopant pair-states can simply be counted; the second method relies on the statistical analysis of a finite crystal. The equations to calculate the probabilities that are shown are valid in any crystal structure. Several examples are shown to illustrate the success of the analysis and to show the influence of the surface to volume ratio on the probabilities.

\section{Simulations and numerical results}

In a recent paper, the simulation algorithm was presented and discussed in detail [12]. ${ }^{2}$ Therefore, only an outline of the simulation method is discussed here. Through the introduction of the

\footnotetext{
${ }^{2}$ Please note that Eq. (5) in that paper is incorrect. It should be replaced by Eq. (4) from this paper, as discussed in Phys. Rev. B 66 (2002) 079901(E).
}

dimensionless radius $\rho \equiv(r / a)$ a formalism is described that does not depend on the lattice parameter $a$ and holds for any crystal with the same crystal structure. The zincblende crystallographic structure is used for the calculations.

For each crystal size and dopant concentration, a set of $1 \times 10^{5}$ nanocrystals is simulated. Each of $n$ lattice positions in the crystal is occupied by a dopant with probability $f$. This results in $k=f n$ dopants in the lattice. A random dopant distribution is assumed (i.e. no preferential pair-formation). Whenever a dopant ion has a dopant as a nearest neighbor, then this is counted as a pair-state. The presence of triple-states, which in the zincblende crystal structure can be three particles on a line (which would count as four pair-states in the algorithm), or three particles forming a triangle (counting as six pair-states), is not taken into account correctly. As a result, the algorithm overestimates the actual number of dopant pair-states in the case of a large dopant fraction. This is done because the combinatorial problems associated with these "higher-order" corrections become increasingly more difficult to handle and require much more computer-time to be correctly evaluated. The probability of finding at least one dopant pair-state in a nanocrystal of size $\rho$ with a dopant fraction $f$ is denoted by $\mathcal{P}(\rho, f)$. The fraction of dopants that are part of a pair-state in the same crystal is denoted by $\mathcal{Q}(\rho, f)$.

Two typical simulations are shown in Fig. 1. These examples were calculated using $\rho=4$ (i.e. $n=1048$ ) and $f=0.01$ (i.e. $k=10$ ). In the simulation on the left, a pair-state has formed as can be seen in the top right part of the simulated crystal. The simulation on the right did not result in a pair-state. For this specific case, values were found of $\mathcal{P}(4,0.01)=0.3921$ and $\mathcal{Q}(4,0.01)=$ $8.8 \%$.

In Fig. 2(a) the probability of finding at least one dopant pair-state in a nanocrystal of size $\rho=6$ is shown. The line through the data and the two dashed lines are related to the theoretical prediction which will be discussed later. Fig. 2(b) clearly shows the strong influence of the crystal size (i.e. the fraction of lattice positions at the 

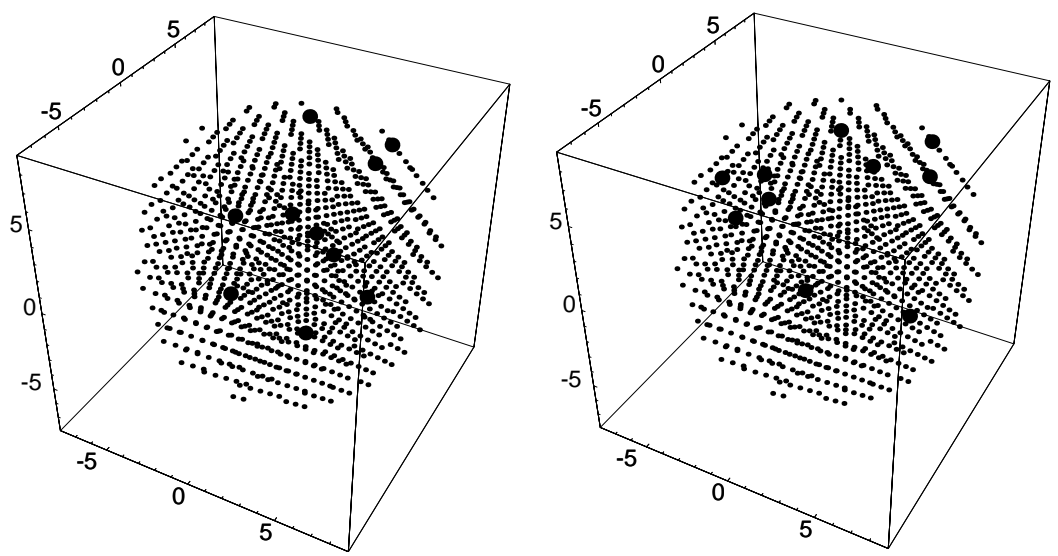

Fig. 1. Two typical simulations obtained for $\rho=4$ and $f=0.01$. The small dots $(\cdot)$ represent the lattice and the large dots $(\bullet)$ indicate the dopants present in this lattice. In the crystal on the left a pair-state is observed (upper right part), whereas in the crystal on the right there is not.
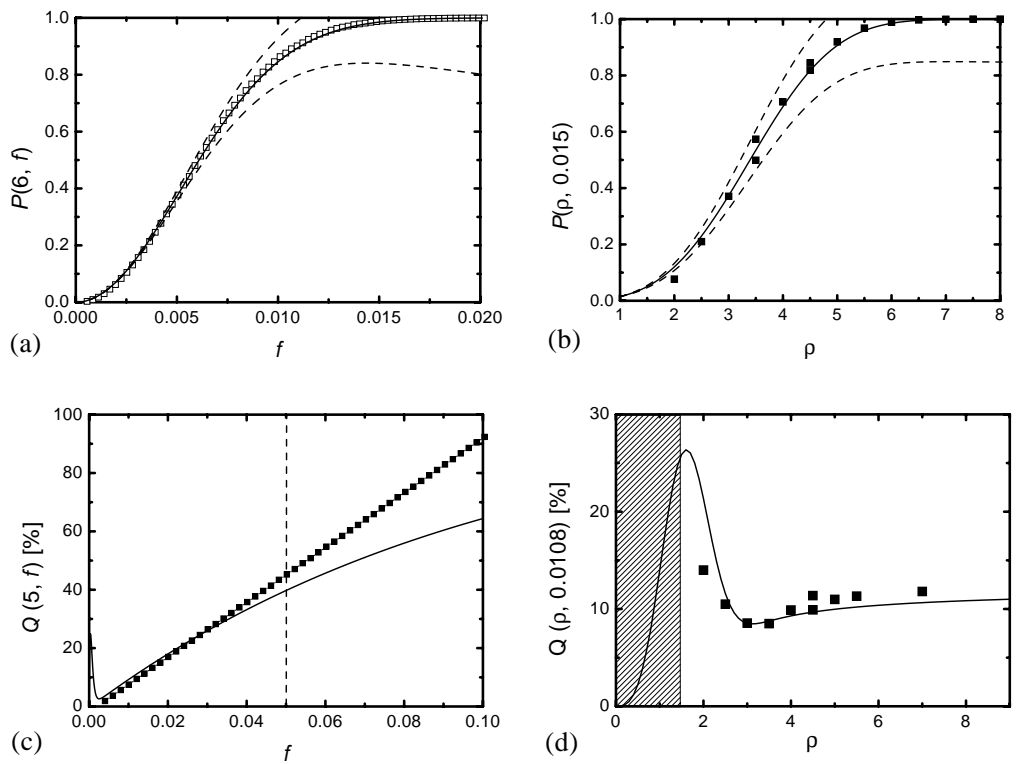

Fig. 2. (a) Probability of finding at least one pair-state in a nanocrystal of size $\rho=6$ as a function of the dopant concentration. (b) The same, but now as a function of the crystal size for fixed dopant concentration $f=0.015$. The lines through the data in (a) and (b) represent the prediction from Eq. (2) based on the Stein-Chen Poisson approximation and the dashed lines were calculated using Eq. (3) and can be thought of as the confidence interval. (c) The percentage of dopants that are part of a pair-state in a nanocrystal of size $\rho=5$ as a function of the dopant concentration. (d) The same, but now the dopant concentration is fixed at $f=0.0108$ and the crystal size is varied. The lines in figures (c) and (d) are the theoretical predictions from Eq. (4).

surface of the nanocrystal) on the total probability of finding at least one dopant pair-state. At two specific crystal sizes, there are two simulation data points per crystal size. For these crystal sizes the concentration $f=0.015$ was not simulated. The data points represent the simulations that were performed with dopant concentrations closest to $f=0.015$ for these crystal sizes. Regardless of the 
ratio of surface to volume lattice positions, the probability of finding at least one pair-state in any nanocrystal will converge to 1 for large crystal sizes. However, the trend shown in Fig. 2(b) converges to 1 faster than expected. This can be seen from the following argument: For $\rho=7$ the surface contribution will be relatively small and the simulation shows $\mathcal{P}(7,0.0052)=0.57$. If the surface did not influence the probability distribution, then $\mathcal{P}(2, f)=0.57$ may be solved through $f=(7 / 2)^{3} \cdot 0.0052=0.22$ because of the cubic dependence of $n$ on $\rho$. However, the simulations show that $\mathcal{P}(2,0.043)=0.57$. The much smaller dopant-fraction required (about $0.23 / 0.043=5$ times) is a direct result of the larger surface contribution to the probability distribution.

Fig. 2(c) shows the percentage of dopants that are part of a pair-state (for fixed $\rho=5$ ) as a function of the dopant concentration. Again, the line is a prediction, which will be discussed later. A comparison of such graphs for different $\rho$ shows clearly the influence of the surface, just as in the previous section. If the surface did not influence the results, then one would expect $\mathcal{Q}(2, f)=$ $\mathcal{Q}(7, f)$ for all $f$. However, the data show that $\mathcal{Q}(2,0.04)=22 \%$, while $\mathcal{Q}(7,0.04)=10 \%$. Again, this difference is explained by the increased probability for pair-state formation of a dopant at the surface of the nanocrystal with respect to a bulk dopant. The straight lines, such as the example shown in Fig. 2(c), do not seem to level off. Indeed for $f>0.12$ in the example shown, it is found that $\mathcal{Q}>100 \%$. This is the result of the presence of triple-states (that allow for three dopants to count as up to six pair-states), which can no longer be ignored. Therefore, the simulations with large dopant fractions (such as $f \approx 0.1$ ) are deemed unrealistic and should be discarded. The reason that the data shown in Fig. 2(c) give a straight line is relatively straightforward. For low-dopant fractions, there are almost no triple-states. Therefore, the number of dopants that are in a pair-state will scale with $f^{2}$ (two dopants need to be next to each other, each with probability $f$ ). As the number of dopants in the lattice scales with $f$, it is clear that the fraction of dopants that are in a pair-state scales with $f^{2} / f=f$.

\section{Mathematical description}

In this section the theoretical predictions for the probability of find at least one pair-state in the crystal, an upper bound for the error introduced in this prediction, and the fraction of dopants that are part of a pair-state are presented. The equations shown are valid in any crystal structure and depend on the values for the number of neighbors of a bulk lattice position (the bulk connectivity, $C_{\mathrm{b}}$ ), the number of neighbors of a surface lattice position (the surface connectivity, $C_{\mathrm{s}}$ ) and the number of lattice positions present in the unit-cell $(\psi)$. A lattice position is defined to be a surface position, denoted as $n_{\mathrm{s}}(\rho)$, if it contributes to $n(\rho)$ but not to $n(\rho-1)$. All other lattice positions, denoted by $n_{\mathrm{b}}(\rho)$, are defined as the bulk lattice positions. The total number of lattice positions in the crystal is approximated by

$n(\rho) \approx \frac{4 \pi \psi}{3} \rho^{3}$.

In this approximation, the number of surface lattice positions is given by $n_{\mathrm{s}}(\rho)=n(\rho)-n(\rho-$ 1) $=4 \pi \psi[1+3 \rho(\rho-1)] / 3$ and the number of bulk lattice positions by $n_{\mathrm{b}}(\rho)=n(\rho-1)=$ $4 \pi \psi(\rho-1)^{3} / 3$. This approximation is extremely successful, even for a very small nanocrystal, such as $\rho=2$ [12].

The method that was used to find the approximation of $\mathcal{P}(\rho, f)$ is a special instance of the SteinChen Poisson approximation method [13]. The main idea is that the number of pair-states is close in distribution to a Poisson random variable. This is due to the fact that the pair-states are almost independent. The number of nearest-neighbor pairs in the crystal is denoted by $N=\frac{1}{2}\left(n_{\mathrm{s}} C_{\mathrm{s}}+\right.$ $n_{\mathrm{b}} C_{\mathrm{b}}$ ). To form a pair-state in the crystal, two nearest-neighbor lattice positions must be occupied by a dopant. The probability that a lattice position contains a dopant is denoted by $f$ and therefore the probability that two lattice positions are occupied by a dopant will be $f^{2}$. As there are $N$ nearest-neighbor pairs in the crystal, the expected number of dopant pair-states will simply be given by $\lambda=N f^{2}$. Based on the above, it can now be proven that the probability of finding at least one 
dopant pair-state can be approximated by ${ }^{3}$

$$
\begin{aligned}
\mathcal{P}= & 1-\exp \left[-\frac{2 \pi \psi f^{2}}{3}\left\{C_{\mathrm{b}}(\rho-1)^{3}\right.\right. \\
& \left.\left.+C_{\mathrm{s}}(3 \rho(\rho-1)+1)\right\}\right],
\end{aligned}
$$

and that the error introduced by this approximation is bounded by (see footnote 3 )

$$
\begin{aligned}
& |\mathcal{P}(\rho, f)-\mathcal{P}| \\
& \leqslant\left(1-\mathrm{e}^{-N f^{2}}\right)\left\{\frac{4 \pi \psi\left(f+f^{2}\right)}{3 N}\right. \\
& \quad \times\left[(\rho-1)^{3} C_{\mathrm{b}}\left(C_{\mathrm{b}}-1\right)\right. \\
& \left.\left.\quad+(1+3 \rho(\rho-1)) C_{\mathrm{s}}\left(C_{\mathrm{s}}-1\right)\right]+f^{2}\right\} .
\end{aligned}
$$

The numbers resulting from Eqs. (2) and (3) can be easily computed, and this means that simulations are no longer needed. As long as the upper bound for the error in $\mathcal{P}$, as found from the evaluation of Eq. (3) remains small, the SteinChen Poisson approximation of $\mathcal{P}$ can be used directly. As an example, consider the simulation data shown in Fig. 1. For $\psi=4, C_{\mathrm{b}}=12$ and $C_{\mathrm{s}}=8$, a simple evaluation for $\rho=4$ and $f=0.01$ leads to $\mathcal{P}=0.405$. This is in very good agreement with the result obtained from the simulation (0.3921). The upper bound for the error introduced in the approximation is also computed, and results in $|\mathcal{P}(\rho, f)-\mathcal{P}|=0.074$. Note that the simulations indicate that the actual error $(0.013)$ is even much smaller than the theoretical upper bound.

Because $\lambda$ represents the (expected) number of dopant pair-states and $k$ represents the number of dopants present in the crystal, it might be expected that the fraction of dopants that are part of a pairstate scales with $\lambda / k$. However, this is not correct. The reason lies in the fact that $\lambda$ is the expectation value for the number of pair states and $k$ is the expectation value for the number of dopants present in the lattice. To find the fraction of dopants that are part of a pair-state, one should calculate the expectation value of $(\lambda / k)$, which is

\footnotetext{
${ }^{3}$ The derivations that led to Eqs. (2), (3) and (4) are quite complicated and would require too much space to be presented here. The interested reader can obtain a copy of the derivation from the authors.
}

different from the expectation value of $\lambda$ divided by the expectation value of $k$ [14]. Using the known forms of $n, n_{\mathrm{s}}$ and $n_{\mathrm{b}}$ (based on the approximation given in Eq. (1)), the expression that gives the fraction of dopants that are part of a pair-state is (see footnote 3 ),

$$
\begin{aligned}
\mathcal{Q}= & \left(1-(1-f)^{4 \pi \psi \rho^{3} / 3}\right)\left[1-\frac{4 \pi \psi(\rho-1)^{3}}{4 \pi \psi \rho^{3}-3 C_{\mathrm{b}}}\right. \\
& \times\left((1-f)^{C_{\mathrm{b}}}-(1-f)^{4 \pi \psi \rho^{3} / 3}\right) \\
& -\frac{4 \pi \psi(1+3 \rho(\rho-1))}{4 \pi \psi \rho^{3}-3 C_{\mathrm{s}}} \\
& \left.\times\left((1-f)^{C_{\mathrm{s}}}-(1-f)^{4 \pi \psi \rho^{3} / 3}\right)\right] .
\end{aligned}
$$

The calculation that led to Eq. (4) is exact: no assumptions are made other than a random dopant concentration in a spherical crystal. As a result, this equation will be valid in any crystal structure, regardless of size and dopant concentration.

\section{Examples}

As a set of typical examples, the probability of forming at least one pair-state and the percentage of pair-states in a $\mathrm{ZnSe}: \mathrm{Mn}^{2+}$ sample have been calculated. The $\mathrm{ZnSe}$ lattice has a zincblende structure $\left(\psi=4, C_{\mathrm{b}}=12\right.$ and $\left.C_{\mathrm{s}}=8\right)$ with a lattice parameter $a=5.6676 \AA$ and the $\mathrm{Mn}^{2+}$ dopant ions are located on the $\mathrm{Zn}^{2+}$ lattice position.

Fig. 2(a) shows the effect of the dopant concentration on the probability of finding at least one pair-state for a nanocrystal of a fixed size $\rho=6$. It can be seen directly from Fig. 2(a) that for $f>0.01$ the upper bound for the error introduced by the Stein-Chen Poisson approximation becomes large (indicated by the dashed lines). However, it is also clear from this figure that the prediction for the probability to find at least one pair-state in the nanocrystal based on this approximation remains very accurate for all dopant concentrations simulated. This proves that the Poisson approximation will also yield reliable data for high dopant fractions.

Fig. 2(b) clearly shows the strong influence of the ratio of surface to volume lattice positions on the 
total probability of finding at least one dopant pairstate. In this figure the dopant fraction in the nanocrystal is fixed at $f=0.015$, while the crystal size is varied. It can be clearly seen from this figure that there is a large influence of the crystal size on the pair formation probability. The line through the data points is a prediction of the simulation data using the Stein-Chen Poisson theory described previously. The prediction from the Stein-Chen Poisson theory gives the exact same trend as the simulation data. The part with $\rho<2$ shows no data points from the simulations because in this range $f$ is so small that $k<2$ (i.e. not enough dopants to form a pair-state). At two specific crystal sizes, there are two simulation data points per crystal size. They represent again the results for the dopant concentrations closest to $f=0.015$, as was explained earlier.

The data shown in Fig. 2(c) provide evidence of the presence of triple-states at high dopant concentrations. In this figure the percentage of dopants that are part of a pair-state is shown as a function of dopant concentration, for nanocrystals with a radius $\rho=5$. The simulation data indicates that $\mathcal{Q}(5, f)$ is linear in $f$. The prediction from Eq. (4) indicates that this is indeed the case for dopant fractions below roughly $5 \%$ (indicated by the dashed line in the figure). For large dopant concentrations, a significant deviation from linearity is observed. Obviously $\mathcal{Q}<\mathcal{Q}(5, f)$ as the calculation that led to Eq. (4) does take triplestates and higher order contributions into account correctly, whereas the simulations are known to overestimate their contribution to the fraction of dopants that are part of a pair-state. Therefore, the fact that for large $f$ the prediction becomes sub-linear is related to the formation of dopant triple-states.

Finally, a very counter-intuitive observation can be drawn from Fig. 2(d), where the percentage of dopants that are part of a pair-state is shown as a function of the crystal size for a fixed dopant concentration $f=0.0108$. It is directly clear from this figure that the concentration does not decrease monotonously for increasing crystal size. The prediction of Eq. (4) agrees rather well with the data and is able to predict the observed trend: As the crystals become larger, the fraction of dopants that are part of a pair-state decreases, until a minimum is reached. From this minimum upward the fraction increases again and converges to the bulk value $(1-f)^{C_{\mathrm{b}}}$. At very small crystal sizes $(\rho \approx 1.5)$ a maximum in $\mathcal{Q}$ is observed. This feature is an artifact of the calculation that led to Eq. (4). For very small crystals, the expected number of dopants goes to zero. Therefore also the number of pair-states goes to zero. As a result, there are no dopants that are part of a pair-state and also no dopants that are isolated. This leads to a contradiction because of the way the expression for $\mathcal{Q}$ has been derived. At this point, Eq. (4) beaks down and can no longer be used (indicated by the dashed box). This is however only a mathematical problem, as the physical situation represents a lattice with no dopant ions. The observant reader can see the same problem in Fig. 2(c) for $f \approx 0$.

It is important to stress that the lines through the simulation data shown in Fig. 2(a)-(d) are not fits, they are theoretical predictions. The excellent agreement between the simulations and the theory shows that the theory is valid in any crystal structure. Therefore, simulations of other types of nanocrystals are not required. All that remains now (in the view of the authors) is an experiment to verify the correctness of Eqs. (2) and (4). For such an experiment, one could think of a rare-earth doped rare-earth oxide nanocrystal, such as $\mathrm{Y}_{2} \mathrm{O}_{3}: \mathrm{Eu}^{3+}$. The reason to use rare-earths is the chemical equivalence of most of the rareearth elements. As a result, preferential pairformation is not expected and Eqs. (2) and (4) should therefore be valid. As the actual number of dopants in a nanocrystal has a binomial distribution, many nanocrystals of identical size and (synthesized) dopant concentration will have to be investigated to obtain the high-quality statistics required.

\section{Conclusions}

This paper presents the results from simulations for determining the probabilities of finding dopant pair-states in a nanocrystal that have general applicability in nanocrystal science. 
The probability of finding at least one pair state and the percentage of dopants that are part of a pair-state are calculated explicitly for a nanocrystal with the zincblende structure. The results are independent of the lattice parameter. Through an approximation, the pair-state probabilities are estimated. A closed-form approximation is presented that is applicable to any nanocrystal, regardless of size and crystallographic structure. These results are valid for any dopant fraction. As a result, the theory is complete and eagerly awaiting experimental verification.

\section{Acknowledgements}

This work is part of the Research Program of the Priority Program for new Materials (PPM) and was made possible by financial support from the Dutch association for scientific research (NWO).

\section{References}

[1] B. Henderson, G.F. Imbusch, Optical Spectroscopy of Inorganic Solids, Clarendon Press, Oxford, 1989 (Chapter $10)$.

[2] R.E. Behringer, J. Chem. Phys. 29 (1958) 537.

[3] R. Rosetti, R. Hull, J.M. Gibson, L.E. Brus, J. Chem. Phys. 82 (1985) 552.

[4] L. Brus, J. Phys. Chem. 90 (1986) 2555.

[5] A. Henglein, Chem. Rev. 89 (1989) 1861.

[6] N.C. Greenham, X. Peng, A.P. Alivisatos, Phys. Rev. B 54 (1996) 17628.

[7] J.F. Suyver, S.F. Wuister, J.J. Kelly, A. Meijerink, Phys. Chem. Chem. Phys. 2 (2000) 5445.

[8] D.G. Thomas, J.J. Hopfield, C.J. Frosch, Phys. Rev. Lett. 15 (1965) 857.

[9] K. Riwotzki, M. Haase, J. Phys. Chem. B 102 (1998) 10129.

[10] C.R. Ronda, T. Amrein, J. Lumin. 69 (1996) 245.

[11] A.A. Maksimov, G. Bacher, et al., Phys. Rev. B 62 (2000) R7767.

[12] J.F. Suyver, R. Meester, J.J. Kelly, A. Meijerink, Phys. Rev. B 64 (2001) 235408.

[13] A.D. Barbour, L. Holst, S. Janson, Poisson Approximation, Clarendon Press, Oxford, 1992.

[14] S. Ross, A First Course in Probability, 6th Edition, Prentice-Hall, New Jersey, 2002 (Chapter 4). 